\title{
Broad geographic, taxonomic and ecological patterns of interpopulation variation in the dietary habits of snakes
}

\author{
Luca Luiselli
}

Luiselli, L. 2006. Broad geographic, taxonomic and ecological patterns of interpopulation variation in the dietary habits of snakes. - Web Ecol. 6: 2-16.

Because of their unique morphological and ecological characteristics (i.e. being obligate carnivorous, solitary, and ingesting their prey whole), snakes are expected to show unusual dietary patterns compared to other ectothermic vertebrates, and the best way to explore this is to analyse the snake dietary patterns globally. Here I review and analyse the peer-reviewed snake diet literature available in order to explore whether there are broad patterns in the interpopulation variability of diet composition in these unique ectothermic predators. I collated data for 181 independent populations belonging to 58 species of snakes from some of the main families (1 Boidae, 2 Pythonidae, 27 Colubridae, 10 Elapidae, and 18 Viperidae) and from all the continents (4 from South and Central America, 13 from North America, 12 from Europe, 18 from Africa, 4 from Asia, and 7 from Australia). All these populations satisfied some precise criteria of inclusion, and were therefore re-analysed in a comparative perspective. I classified each literature entry according to 1) snake species, 2) snake family, 3) geographic position (continent) of the study areas, 4) climatic region (temperate versus tropical), 5) guild (if the species is aquatic, terrestrial, or arboreal), 6) hunting strategy (sit-and-wait versus active forager), and 7) venom (if the species is venomous or not). All these seven factors were analysed by GLM procedures to evaluate their effects on the interpopulation diet variation within snake species, that was assessed by using a univariate similarity index. The various taxonomical categories of snake prey were grouped according to two different levels of taxonomic affinity: a) general affinity, e.g. frogs and toads, salamanders, lizards, birds, etc., and b) close affinity, by grouping prey types belonging to a same genus. My study revealed that, within-species snake populations showed a very low variability in terms of diet composition. As for the general affinity criterion, there was no single factor that produced a significant effect on the interpopulation diet variation but, with regard to the interaction terms between factors, significant effects were determined by 1) continent $\times$ climatic region (with Asian, African and South-American tropical populations having significantly lesser similarity values), 2) continent $\times$ hunting strategy (diets being less diverse in ambush predators in Africa, Asia and South America), and 3) climatic region $\times$ guild (with arboreal tropical snakes showing less interpopulation similarity). As for the close affinity criterion, there was also no effect of single factors on interpopulation diet variability, but the interaction term hunting strategy $\times$ venom was significant, with sit-and-wait venomous species being less variable in their diet composition. Snake family was completely un-influent in determining any effect on snake interpopulation diet variation. The broad reasons that may explain these generalized patterns are discussed.

L. Luiselli (lucamlu@tin.it), FI.Z.V. (Ecology), and Centre of Environmental Studies "Demetra" s.r.l., via Olona 7, IT-00198 Rome, Italy. 
The study of the feeding habits of animals has attracted much attention by ecologists, and thousands of contributions have appeared during the recent years, either theoretical (Schoener 1971, Stephens and Krebs 1986, Green 2006, Olsson and Brown 2006, etc.) or empirical (Ray and Sunquist 2001, Lekunze et al. 2001, and lots of other studies). As a result, we currently do know much more than just a few decades ago about the diet composition, and its ecological and behavioural determinants, of a number of living animals belonging to different taxonomic groups (for a case study, see Carretero 2004).

Snakes are unique among vertebrates because of some peculiar adaptations: they are exclusively carnivorous and solitary (but see Shine et al. 2002), and obligatorily ingest their prey whole (Greene 2001). Thus, they are gape-limited, and have evolved a suite of morphological and behavioural adaptations to kill their prey and ingest it whole, including sophisticated venom and the apparatus to inject it, extreme body strength and huge size to suffocate very large animals, and enormous skull distensibility (Greene 2001). Because of these unique characteristics, these animals can be expected to show unusual dietary patterns compared to other ectothermic vertebrates, and the best way to explore this is to analyse the snake dietary patterns globally, given that the use of specific study models may be partially inadequate to understand broader patterns. Up to the early 1990s, snakes remained relatively unstudied, but currently they have achieved the status of "model organisms" for ecological and evolutionary studies, and hence several broad studies have been published (Shine 1991, Shine and Bonnet 2000, Luiselli 2006a).

Although the study of the diet composition of freeranging snakes has traditionally played a major argument of research for snake ecologists (Mushinsky 1987), nonetheless there is still no published attempt at identifying the broad patterns (geographic, taxonomic, and ecological) of the interpopulation variation within the snake species' dietary habits. This is a important shortcoming for our understanding of snake evolution and ecology, also in consideration of the fact that previous studies evidenced that dietary habits have crucial relevance in determining the morphological traits of snakes (for instance, the sexual size dimorphism and the head size and shape: Shine 1991, Shetty and Shine 2002), and that snakes are unusual among carnivorous vertebrates because they usually partition the food resource and not the spatial resource when in competition (Luiselli 2006a). The study of the interpopulation snake diet variation may be important to understand whether there are broad unifying patterns (at the taxonomical, geographical, or eco-ethological level) that may explain patterns and correlates of dietary variation, and this may in turn be useful also for comparisons with other carnivorous vertebrates in order to learn more of the evolution of the predatory behaviour in animals.

It therefore seems necessary to review recently published material on the interpopulation diet variation in snakes, and to respond to the following questions: 1) do the snakes species vary remarkably between populations in terms of their diet composition? 2) What factors are associated to the interpopulation variation in diet composition: systematic position (i.e. family), geographic origin (i.e. continent), climatic conditions (i.e. temperate versus tropical), ecological guild (i.e. terrestrial, aquatic or arboreal), hunting strategy (i.e. sit-and-wait versus active foraging), or presence/absence of venom apparatus? In this regard, it should however be noticed that several snakes exhibiting one of these factors also may exhibit the other one; i.e. several snakes that practice the sit-and-wait foraging strategy are also venomous (for instance, Bitis gabonica and Bitis nasicornis, see Luiselli 2006a). 3) Which reasons can explain the eventual observed patterns?

I will demonstrate that the impetuous advance of our understanding of snake ecology (Shine and Bonnet 2000) may prove useful to solve the above-mentioned issues, although many snake studies, because of the intrinsic elusiveness of the subject animals, still focus on just documenting small samples of food items taken from free-ranging snakes.

\section{Materials and methods}

I have reviewed through very extensive searches of the literature, the available data on the dietary habits of snake populations around the globe. Only those studies that furnished re-analysable raw data were selected for further inspection. I then considered for the proper statistical study only those bibliographic entries which met with the following conditions: 1 ) they should report precise diet data lists for distinct populations of snakes (Luiselli et al. 1996, 1997, Gregory and Isaac 2004), or diet data lists from snakes occurring in well-defined and habitat-homogenous geographically restricted regions (Shine 1987a, b, c, 1989, etc.); 2) they should report diet records gotten from at least 30 different snake specimens of each population. Studies reporting smaller sample sizes, albeit solid, were not considered for this study (e.g. some data in Shine 1981, 1982, Reynolds and Scott 1982). Although my search for appropriate literature datasets is likely not exhaustively complete, nonetheless it seems to be adequate for the scopes of this paper, given that it collates data from a wide range of different snake genera belonging to a wide range of families in a wide range of geographic and environmental contexts.

Only studies from peer-reviewed journals or, exceptionally, academic dissertations, were used. Every effort was made to avoid unbalanced coverage of the literature from the different regions of the world/snake family/snake genera, although where there is a difference in the amount of literature reviewed this reflects differences in the development of research on snake feeding ecology. For instance, some countries (e.g. USA, Italy, Nigeria, Australia) were 
disproportionately over-represented in my review. I confined my review only to the interpopulation variation in the taxonomic composition of the diet, as no adequate comparative dataset is available for other dietary aspects such as, e.g. prey-size, prey-size-predator-size relationships, etc.

The various taxonomical categories of snake prey were grouped according to two different levels of taxonomic affinity (defined affinity criteria in the following text): a) general affinity (e.g. frogs and toads, salamanders, lizards, birds, etc.), and b) close affinity, by grouping prey types belonging to a same genus (e.g. Triturus species, Rattus species, etc.). I avoided to consider also the interpopulation variation at the prey-species level because there are several cases in which snake populations may actually prey on a same type of prey (for instance, Podarcis species in the case of Coronella girondica), but on different species simply because different species may occur in the different areas where the snake diets were studied (for instance, P. hispanica in Spanish Coronella girondica, and P. sicula in the case of Italian Coronella girondica; Luiselli et al. 2001a). Hence, the actual interpopulation variation may be unrealistically overestimated by using species-level differences in prey type categories.

Similarity in prey types between different populations of each species were estimated by applying Pianka’s (1986) symmetric equation of overlap. Although Pianka’s overlap formula was originarily designed to assess niche overlap between two potential competitors, nonetheless it is merely a similarity index, and hence can be readily used as a univariate measure of similarity in dietary spectrum between two different populations of animals (Pianka 1986). By this formula, the values ranged from 0 (no similarity) to 1 (total identity). This formula is very appropriate for this study, because Pianka's index is similar to MacArthur and Levins (1967) index, with the denominator that has been normalized to make it symmetric, but the stability properties were unchanged (May 1975). For each species, I have built a data matrix in which each cell was the Pianka's similarity index between two populations (see Table 1 for an example), and the various matrices varied in size from a single cell (when data from just two populations of a single population are available) to 28 cells (when data from 8 different populations are available; Table 1). Then, I calculated the mean similarity index of each species by calculating the arithmetic mean of the pairwise similarity indexes.

Each literature entry was classified according to 1) snake species, 2) snake family, 3) geographic position of the study areas (at a continental level: Europe, North America, South and Central America, Africa, Asia, Australia), 4) climatic region (i.e. temperate versus tropical), 5)

Table 1. Example of a data matrix used for this study; the species used for this example is Natrix natrix. Each cell in this matrix represents the value of Pianka's (1986) similarity index between two populations of the study species; also the study areas and the original literature sources are given. Note that this dataset is relative to diet similarity expressed as "general affinity" (see the text for more details).

\begin{tabular}{|c|c|c|c|c|c|c|c|}
\hline & $\begin{array}{l}\text { England } \\
\text { (Stebbings in } \\
\text { Gregory and } \\
\text { Isaac 2004) }\end{array}$ & $\begin{array}{l}\text { England } \\
\text { (Reading and } \\
\text { Davies 1996) }\end{array}$ & $\begin{array}{l}\text { Sweden } \\
\text { (Madsen } \\
1983)\end{array}$ & $\begin{array}{l}\text { Italy } \\
\text { (Luiselli and } \\
\text { Rugiero 1991) }\end{array}$ & $\begin{array}{l}\text { Italy } \\
\text { (Filippi } \\
\text { et al. 1995) }\end{array}$ & $\begin{array}{l}\text { Italy } \\
\text { (Luiselli } \\
\text { et al. 1997) }\end{array}$ & $\begin{array}{l}\text { Italy } \\
\text { (Luiselli } \\
\text { et al. 2005) }\end{array}$ \\
\hline $\begin{array}{l}\text { England } \\
\text { (Gregory and } \\
\text { Isaac 2004) }\end{array}$ & 0.9418 & 0.9299 & 0.9286 & 0.9321 & 0.9293 & 0.9351 & 0.9003 \\
\hline $\begin{array}{l}\text { England } \\
\text { (Stebbings in } \\
\text { Gregory and } \\
\text { Isaac 2004) }\end{array}$ & & 0.9981 & 0.9966 & 0.9983 & 0.9983 & 0.9956 & 0.7036 \\
\hline $\begin{array}{l}\text { England } \\
\text { (Reading and } \\
\text { Davies 1996) }\end{array}$ & & & 0.9961 & 0.9985 & 0.9874 & 0.9909 & 0.6797 \\
\hline $\begin{array}{l}\text { Sweden } \\
\quad \text { (Madsen 1983) }\end{array}$ & & & & 0.9992 & 0.9969 & 0.9871 & 0.6771 \\
\hline $\begin{array}{l}\text { Italy } \\
\quad \text { (Luiselli and } \\
\quad \text { Rugiero 1991) }\end{array}$ & & & & & 0.9943 & 0.9897 & 0.6826 \\
\hline $\begin{array}{l}\text { Italy } \\
\qquad \text { (Filippi } \\
\text { et al. 1995) }\end{array}$ & & & & & & 0.9794 & 0.6852 \\
\hline $\begin{array}{l}\text { Italy } \\
\qquad \text { (Luiselli } \\
\text { et al. 1997) }\end{array}$ & & & & & & & 0.7008 \\
\hline
\end{tabular}


guild (i.e. if the species is aquatic, terrestrial, or arboreal, see Luiselli 2006a), 6) hunting strategy (i.e. if the species is a sit-and-wait or a active forager), and 7) venom (i.e. if the species is venomous and use its venom for killing its prey or not). All these seven factors were analysed to evaluate their effects on the interpopulation diet variation within snake species.

All statistics were done by a SPSS (ver. 11.0) PC package, with all tests being two-tailed and alpha set at $5 \%$. The effects of the seven above-mentioned factors, entered singly in the analysis, on the snake interpopulation diet overlap values, were tested by means of one-way ANOVAs, since the assumptions of normality and homoscedasticity were met. To evaluate the effects of the interaction terms between the various factors on the snake interpopulation diet overlap values, I fitted a generalized linear model (McCullagh and Nelder 1989) using the general factorial design in SPSS, followed by Tukey's post-hoc test, since logtransformed data satisfied the normality and homoscedasticity assumptions. All possible two-way and threeway interactions among the seven factors were included as fixed-effect factors in the model (McCullagh and Searle 2002).

\section{Confounding factors of the analysis}

I identified the following confounding factors for my analysis: 1) different methods used to collect data (i.e. only stomach contents or stomach+gut contents; dissection of museum vouchers or examination of free-living specimens by abdominal palpation. Unfortunately, no study has examined the possible discrepancies in the description of snake diets by considering only stomach or stomach + gut food contents, thus it is impossible to determine whether this factor may have or may have not biased the analyses. 2) Unstandardized mean distance between study areas within- and among-species. It is possible that the species whose the study populations came from greater distances differed more than those whose study populations were geographically closer. However, given the still fragmentary knowledge on the study subject, it is impossible to assess properly whether or not this factor may have partially biased the analyses. 3) Many snakes show remarkable ontogenetic dietary changes (Saint Girons 1980, Luiselli and Agrimi 1991) and intersexual dietary divergence (Shine 1991). These changes may bias the comparative analyses, so I considered when possible 1) only the adults and 2) assumed that, on average, males and females contributed equally to the examination of the diet of a given snake composition, thus considering males + females data pooled. This latter point is certainly correct, given that all authors tended to present diet data well equilibrated between sexes. 4) In several cases, it was impossible to calculate the overlap values for the close affinity criterion from the original sources.

\section{Results}

\section{General affinity}

In total, I found re-analyzable data for 181 independent populations belonging to 58 species of snakes from all the main families (1 Boidae, 2 Pythonidae, 27 Colubridae, 10 Elapidae, and 18 Viperidae) and from all the continents (4 from South and Central America, 13 from North America, 12 from Europe, 18 from Africa, 4 from Asia, and 7 from Australia) (Table 2). The great majority of the species showed very high interpopulation diet similarity values when considering the general affinity criterion (mean $\pm \mathrm{SD}=0.885 \pm 0.117$, range $=0.432-0.999, \mathrm{n}=$ 58; Fig. 1).

The means and dispersion measures of the interpopulation diet similarity values divided by continent, guild, and family are given in Fig. 2. Univariate Analysis of Variance models (i.e. 1-way ANOVAs for measuring the effects of single factors, and GLM - general factorial design for the effects of the interaction terms between factors) showed that (Table 3): 1) there was no single factor that produced a significant effect on the interpopulation diet variation; 2) with regard to the interaction terms between factors, the significant effects on the interpopulation diet similarity values were: a) continent $\times$ climatic region (Tukey HSD post-hoc test revealing that Asian, African and SouthAmerican tropical populations had significantly lesser similarity values; in all cases, $\mathrm{p}<0.01$ ), b) continent $\times$ hunting strategy (diets less different in ambush predators in Africa, Asia and South America), c) climatic region $\times$ guild (with arboreal tropical snakes showing less interpopulation similarity in prey composition).

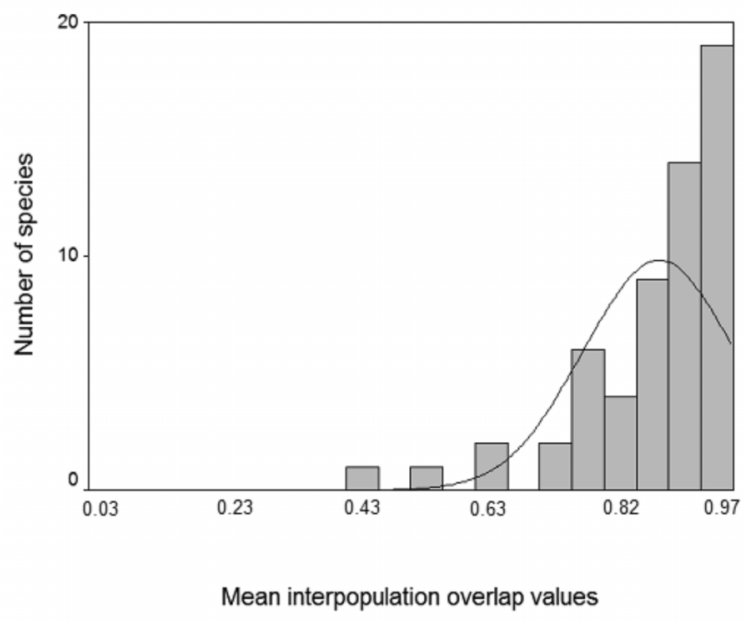

Fig. 1. Distribution of the number of snake species in terms of their interpopulation diet overlap values (calculated by Pianka's formula; all snake families included) as regards the general affinity criterion. The line indicates the normal curve. Note that the great majority of the species are distributed in the very high overlap values intervals (mean $\pm S D=0.885 \pm 0.117, \mathrm{n}=58$ ). 
Table 2. Summary of the interpopulation variation in snake dietary composition, assessed by Pianka's (1986) similarity equation. Symbols: $\mathrm{r}=$ range; $\mathrm{x}=$ mean; $\mathrm{n}=$ number of populations examined.

\begin{tabular}{|c|c|c|c|c|c|}
\hline Species & $\begin{array}{l}\text { Geographic } \\
\text { origin of } \\
\text { the study } \\
\text { populations }\end{array}$ & Main preys & $\begin{array}{l}\text { Similarity (general } \\
\text { affinity) }\end{array}$ & $\begin{array}{l}\text { Similarity (close } \\
\text { affinity) }\end{array}$ & References \\
\hline \multicolumn{6}{|l|}{ Boidae } \\
\hline Boa constrictor & $\begin{array}{l}\text { Argentina, } \\
\text { West Indies }\end{array}$ & $\begin{array}{l}\text { Mammals and birds } \\
\text { (all populations) }\end{array}$ & $0.795 ; \mathrm{n}=2$ & $0.206 ; n=2$ & $\begin{array}{l}\text { Sironi et al. 2000, } \\
\text { Quick et al. 2005 }\end{array}$ \\
\hline \multicolumn{6}{|l|}{ Pythonidae } \\
\hline Python regius & $\begin{array}{l}\text { Benin, } \\
\text { Nigeria }\end{array}$ & $\begin{array}{l}\text { Mammals (all } \\
\text { populations) }\end{array}$ & $0.798 ; n=2$ & $0.463 ; n=2$ & $\begin{array}{l}\text { Politano 1985, Luiselli } \\
\text { and Angelici } 1998\end{array}$ \\
\hline Python sebae & Nigeria & $\begin{array}{l}\text { Mammals (all } \\
\text { populations) }\end{array}$ & $0.776 ; n=2$ & $0.346 ; \mathrm{n}=2$ & Luiselli et al. 2001b \\
\hline \multicolumn{6}{|l|}{ Colubridae } \\
\hline Afronatrix anoscopus & $\begin{array}{l}\text { Benin, } \\
\text { Nigeria }\end{array}$ & $\begin{array}{l}\text { Frogs, tadpoles and } \\
\text { fish (all populations) }\end{array}$ & $\begin{array}{l}r=0.864-0.998 \\
x=0.931 ; n=4\end{array}$ & $\begin{array}{l}\text { Not calculable from } \\
\text { the original datasets }\end{array}$ & $\begin{array}{l}\text { Politano } 1985,1998, \\
\text { Luiselli et al. } 1998 \text { b, } \\
\text { Luiselli } 2006 \mathrm{~b}\end{array}$ \\
\hline Coluber constrictor & USA & $\begin{array}{l}\text { Insects and rodents } \\
\text { (all populations) }\end{array}$ & $\begin{array}{l}r=0.988-0.998 \\
x=0.992 ; n=3\end{array}$ & $\begin{array}{l}\text { Not calculable from } \\
\text { the original datasets }\end{array}$ & $\begin{array}{l}\text { Brown and Parker } \\
\text { 1982, Fitch 1982, } \\
\text { Cooper et al. } 2000\end{array}$ \\
\hline Coronella austriaca & Italy & $\begin{array}{l}\text { Lizards (all } \\
\text { populations) }\end{array}$ & $0.999 ; \mathrm{n}=2$ & $0.163 ; \mathrm{n}=2$ & $\begin{array}{l}\text { Rugiero et al. } 1995 \text {, } \\
\text { Luiselli et al. } 1996\end{array}$ \\
\hline Coronella girondica & $\begin{array}{l}\text { Italy, } \\
\text { Spain }\end{array}$ & $\begin{array}{l}\text { Lizards (all } \\
\text { populations) }\end{array}$ & $0.939 ; \mathrm{n}=2$ & $0.382 ; \mathrm{n}=2$ & Luiselli et al. 2001a \\
\hline Elaphe quadrivirgata & Japan & $\begin{array}{l}\text { Lizards or frogs } \\
\text { (variable depending } \\
\text { on the populations) }\end{array}$ & $\begin{array}{l}r=0.243-0.723 \\
x=0.436 ; n=4\end{array}$ & $\begin{array}{l}\text { Not calculable from } \\
\text { the original datasets }\end{array}$ & $\begin{array}{l}\text { Fukada } 1959,1992, \\
\text { Kadowaki 1992, } \\
\text { 1996, Tanaka and } \\
\text { Ota } 2002\end{array}$ \\
\hline Elaphe quatuorlineata & Italy & $\begin{array}{l}\text { Birds, rodents (all } \\
\text { populations) }\end{array}$ & $\begin{array}{l}\mathrm{r}=0.715-0.973 \\
\mathrm{x}=0.863 ; \mathrm{n}=5\end{array}$ & $\begin{array}{l}r=0.209-0.864 \\
x=0.563 ; n=5\end{array}$ & Filippi et al. 2005 \\
\hline Gastropyxis smaragdina & Nigeria & $\begin{array}{l}\text { Lizards and frogs } \\
\text { (all populations) }\end{array}$ & $0.913, \mathrm{n}=2$ & $0.663 ; \mathrm{n}=2$ & $\begin{array}{l}\text { Luiselli et al. 2000, } \\
\text { Inyang } 2005\end{array}$ \\
\hline $\begin{array}{l}\text { Grayia smythii } \\
\text { (= smithii) }\end{array}$ & Nigeria & $\begin{array}{l}\text { Frogs and fish } \\
\text { (all populations) }\end{array}$ & $0.848 ; n=2$ & $0.863, \mathrm{n}=2$ & $\begin{array}{l}\text { Akani and Luiselli } \\
2001+\text { unpubl., } \\
\text { Luiselli 2006b }\end{array}$ \\
\hline Hemorrhois hippocrepis & $\begin{array}{l}\text { Morocco, } \\
\text { Spain }\end{array}$ & $\begin{array}{l}\text { Rodents, lizards } \\
\text { (all populations) }\end{array}$ & $0.940 ; n=2$ & $0.708 ; n=2$ & $\begin{array}{l}\text { Pleguezuelos and } \\
\text { Moreno 1990, } \\
\text { Pleguezuelos and } \\
\text { Fahd 2004) }\end{array}$ \\
\hline Hierophis viridiflavus & Italy & $\begin{array}{l}\text { Lizards, and rodents } \\
\text { as secondary prey } \\
\text { (all populations) }\end{array}$ & $\begin{array}{l}r=0.942-0.977 \\
x=0.960 ; n=3\end{array}$ & $\begin{array}{l}\mathrm{r}=0.747-0.957 \\
\mathrm{x}=0.841 ; \mathrm{n}=3\end{array}$ & $\begin{array}{l}\text { Rugiero and Luiselli } \\
\text { 1995, Capizzi and } \\
\text { Luiselli 1996, Rugiero } \\
\text { et al. } 2002\end{array}$ \\
\hline Lamprophis fuliginosus & $\begin{array}{l}\text { Benin, } \\
\text { Nigeria }\end{array}$ & $\begin{array}{l}\text { Rodents (all } \\
\text { populations) }\end{array}$ & $\begin{array}{l}r=0.732-0.981 \\
x=0.848 ; n=4\end{array}$ & $\begin{array}{l}\mathrm{r}=0.399-0.901 \\
\mathrm{x}=0.624 ; \mathrm{n}=4\end{array}$ & $\begin{array}{l}\text { Politano } 1985,1998, \\
\text { Inyang } 2005\end{array}$ \\
\hline Natriciteres fuliginoides & Nigeria & $\begin{array}{l}\text { Earthworms and } \\
\text { anuran larvae or } \\
\text { anuran larvae and } \\
\text { metamorphs } \\
\text { (depending on the } \\
\text { populations) }\end{array}$ & $\begin{array}{l}r=0.580-0.988 \\
x=0.787 ; n=4\end{array}$ & $\begin{array}{l}r=0.457-0.936 \\
x=0.644 ; n=4\end{array}$ & Luiselli 2003 \\
\hline Natriciteres variegata & Nigeria & $\begin{array}{l}\text { Amphibian larvae } \\
\text { and metamorphs } \\
\text { (all populations) }\end{array}$ & $0.965 ; n=2$ & $0.965 ; n=2$ & Luiselli 2003 \\
\hline
\end{tabular}


Table 2. Continued.

\begin{tabular}{|c|c|c|c|c|c|}
\hline Species & $\begin{array}{l}\text { Geographic } \\
\text { origin of } \\
\text { the study } \\
\text { populations }\end{array}$ & Main preys & $\begin{array}{l}\text { Similarity (general } \\
\text { affinity) }\end{array}$ & $\begin{array}{l}\text { Similarity (close } \\
\text { affinity) }\end{array}$ & References \\
\hline Natrix maura & $\begin{array}{l}\text { Italy, } \\
\text { Spain }\end{array}$ & $\begin{array}{l}\text { Frogs, or fish and } \\
\text { frogs (depending } \\
\text { on the population) }\end{array}$ & $\begin{array}{l}r=0.140-0.918 \\
x=0.525 ; n=3\end{array}$ & $\begin{array}{l}r=0.006-0.206 \\
x=0.125 ; n=3\end{array}$ & $\begin{array}{l}\text { Santos and Llorente } \\
1998 \text {, Rugiero et al. } \\
2000\end{array}$ \\
\hline Natrix natrix & $\begin{array}{l}\text { England, } \\
\text { Italy, } \\
\text { Sweden }\end{array}$ & $\begin{array}{l}\text { Frogs and toads } \\
\text { (all populations) }\end{array}$ & $\begin{array}{l}\mathrm{r}=0.677-0.999 \\
\mathrm{x}=0.872 ; \mathrm{n}=8\end{array}$ & $\begin{array}{l}r=0.188-0.812 \\
\mathrm{x}=0.472 ; \mathrm{n}=7\end{array}$ & $\begin{array}{l}\text { Madsen 1983, Luiselli } \\
\text { and Rugiero 1991, } \\
\text { Reading and Davies } \\
\text { 1996, Luiselli et al. } \\
\text { 1997, 2005, Gregory } \\
\text { and Isaac } 2004\end{array}$ \\
\hline Natrix tessellata & $\begin{array}{l}\text { Austria, } \\
\text { Italy }\end{array}$ & Fish (all populations) & $\begin{array}{l}r=0.961-0.998 \\
x=0.987 ; n=3\end{array}$ & $\begin{array}{l}r=0.921-0.967 \\
x=0.947 ; n=3\end{array}$ & $\begin{array}{l}\text { Luiselli and Rugiero } \\
\text { 1991, Filippi } 1995, \\
\text { Zimmermann and } \\
\text { Fachbach } 1996\end{array}$ \\
\hline Nerodia sipedon & USA & Fish (all populations) & $0.985, \mathrm{n}=2$ & $0.155, \mathrm{n}=2$ & King 1993 \\
\hline Nerodia taxispilota & USA & Fish (all populations) & $\begin{array}{l}\mathrm{r}=0.912-0.990 \\
\mathrm{x}=0.954 ; \mathrm{n}=3\end{array}$ & $\begin{array}{l}\mathrm{r}=0.548-0.913 \\
\mathrm{x}=0.689 ; \mathrm{n}=3\end{array}$ & $\begin{array}{l}\text { Camp et al. } 1980 \text {, } \\
\text { Gibbons and Dorcas } \\
2004\end{array}$ \\
\hline Pituophis catenifer & USA & $\begin{array}{l}\text { Mammals (all } \\
\text { populations) }\end{array}$ & $\begin{array}{l}\mathrm{r}=0.489-0.951 \\
\mathrm{x}=0.783 ; \mathrm{n}=4\end{array}$ & $\begin{array}{l}\text { Not calculable from } \\
\text { the original datasets }\end{array}$ & $\begin{array}{l}\text { Rodriguez-Robles } \\
2002\end{array}$ \\
\hline Pituophis melanoleucus & USA & $\begin{array}{l}\text { Rodents (all } \\
\text { populations) }\end{array}$ & $0.933 ; \mathrm{n}=2$ & $\begin{array}{l}\text { Not calculable from } \\
\text { the original datasets }\end{array}$ & $\begin{array}{l}\text { Brown and Parker } \\
\text { 1982, Fitch } 1999\end{array}$ \\
\hline Psammophis phillipsii & Nigeria & $\begin{array}{l}\text { Lizards (all } \\
\text { populations) }\end{array}$ & $\begin{array}{l}\mathrm{r}=0.833-0.975 \\
\mathrm{x}=0.891 ; \mathrm{n}=3\end{array}$ & $\begin{array}{l}r=0.439-0.811 \\
x=0.695 ; n=3\end{array}$ & $\begin{array}{l}\text { Politano } 1985 \text {, Akani } \\
\text { et al. } 2003 \text {, Luiselli et } \\
\text { al. } 2004 \mathrm{a}\end{array}$ \\
\hline Rhabdophis tigrinum & Japan & Amphibians & $\begin{array}{l}r=0.865-0.967 \\
x=0.921 ; n=3\end{array}$ & $\begin{array}{l}\text { Not calculable from } \\
\text { the original datasets }\end{array}$ & $\begin{array}{l}\text { Fukada 1959, 1992, } \\
\text { Kadowaki 1992, } \\
\text { Hirai } 2004\end{array}$ \\
\hline Rhamnophis aethiopissa & $\begin{array}{l}\text { Cameroon, } \\
\text { Nigeria }\end{array}$ & $\begin{array}{l}\text { Frogs, or lizards and } \\
\text { birds (depending on } \\
\text { the populations) }\end{array}$ & $\begin{array}{l}\mathrm{r}=0.667-0.811 \\
\mathrm{x}=0.745 ; \mathrm{n}=3\end{array}$ & $\begin{array}{l}r=0.339-0.611 \\
x=0.498 ; n=3\end{array}$ & $\begin{array}{l}\text { Luiselli et al. 1999- } \\
\text { 2000, Inyang } 2005\end{array}$ \\
\hline Thamnophis elegans & $\begin{array}{l}\text { Canada, } \\
\text { USA }\end{array}$ & $\begin{array}{l}\text { Slugs, fish and } \\
\text { amphibians } \\
\text { (depending on } \\
\text { the populations) }\end{array}$ & $\begin{array}{l}r=0.645-0.838 \\
x=0.739 ; n=4\end{array}$ & $\begin{array}{l}\text { Not calculable from } \\
\text { the original datasets }\end{array}$ & $\begin{array}{l}\text { Fitch } 1940 \text {, Fox } 1952 \text {, } \\
\text { Gregory } 1978, \\
\text { Gregory and Nelson } \\
1991\end{array}$ \\
\hline Thamnophis sirtalis & $\begin{array}{l}\text { Canada, } \\
\text { USA }\end{array}$ & $\begin{array}{l}\text { Amphibians and } \\
\text { earthworms (all } \\
\text { populations) }\end{array}$ & $0.919 ; \mathrm{n}=2$ & $\begin{array}{l}\text { Not calculable from } \\
\text { the original datasets }\end{array}$ & $\begin{array}{l}\text { Fitch } 1965,1982, \\
\text { Gregory } 1978\end{array}$ \\
\hline Toxicodryas blandingii & $\begin{array}{l}\text { Benin, } \\
\text { Nigeria }\end{array}$ & $\begin{array}{l}\text { Birds and lizards } \\
\text { (all populations) }\end{array}$ & $\begin{array}{l}r=0.793-0.988 \\
x=0.902 ; n=3\end{array}$ & $\begin{array}{l}r=0.441-0.776 \\
x=0.612 ; n=3\end{array}$ & $\begin{array}{l}\text { Politano } 1985 \text {, Luiselli } \\
\text { et al. 1998a, Inyang } \\
2005\end{array}$ \\
\hline Zamenis longissimus & Italy & $\begin{array}{l}\text { Rodents (all } \\
\text { populations) }\end{array}$ & $\begin{array}{l}\mathrm{r}=0.858-0.998 \\
\mathrm{x}=0.907 ; \mathrm{n}=4\end{array}$ & $\begin{array}{l}r=0.761-0.937 \\
x=0.818 ; n=4\end{array}$ & $\begin{array}{l}\text { Capula and Luiselli } \\
\text { 2002, Rugiero et al. } \\
2002\end{array}$ \\
\hline \multicolumn{6}{|l|}{ Elapidae } \\
\hline Cryptophis nigrescens & Australia & $\begin{array}{l}\text { Lizards (all } \\
\text { populations) }\end{array}$ & $0.998 ; n=2$ & $0.975 ; n=2$ & Shine 1984 \\
\hline Demansia psammophis & Australia & $\begin{array}{l}\text { Lizards (all } \\
\text { populations) }\end{array}$ & $0.997 ; \mathrm{n}=2$ & $0.776 ; n=2$ & Shine 1980 \\
\hline Dendroaspis jamesoni & Nigeria & $\begin{array}{l}\text { Birds and rodents } \\
\text { (all populations) }\end{array}$ & $0.986 ; n=2$ & $0.776 ; n=2$ & $\begin{array}{l}\text { Luiselli et al. 2000b, } \\
\text { Inyang } 2005\end{array}$ \\
\hline Hemiaspis signata & Australia & $\begin{array}{l}\text { Frogs and lizards } \\
\text { (all populations) }\end{array}$ & $0.989 ; n=2$ & $0.870 ; \mathrm{n}=2$ & Shine $1987 \mathrm{a}$ \\
\hline
\end{tabular}


Table 2. Continued.

\begin{tabular}{|c|c|c|c|c|c|}
\hline Species & $\begin{array}{l}\text { Geographic } \\
\text { origin of } \\
\text { the study } \\
\text { populations }\end{array}$ & Main preys & $\begin{array}{l}\text { Similarity (general } \\
\text { affinity) }\end{array}$ & $\begin{array}{l}\text { Similarity (close } \\
\text { affinity) }\end{array}$ & References \\
\hline Naja melanoleuca & Nigeria & $\begin{array}{l}\text { Mammals, } \\
\text { amphibians, } \\
\text { fish }\end{array}$ & $0.819 ; \mathrm{n}=2$ & $0.624 ; \mathrm{n}=2$ & Luiselli et al. 2002 \\
\hline Naja nigricollis & Nigeria & Lizards and mammals & $0.929 ; n=2$ & $0.588 ; \mathrm{n}=2$ & Luiselli et al. 2002 \\
\hline Notechis scutatus & Australia & $\begin{array}{l}\text { Frogs, or frogs and } \\
\text { lizards, or small } \\
\text { mammals (depending } \\
\text { on the populations) }\end{array}$ & $\begin{array}{l}\mathrm{r}=0.468-0.831 \\
\mathrm{x}=0.601 ; \mathrm{n}=3\end{array}$ & $\begin{array}{l}r=0.294-0.335 \\
x=0.316 ; n=3\end{array}$ & Shine $1987 b$ \\
\hline Pseudechis australis & Australia & $\begin{array}{l}\text { Lizards, frogs and } \\
\text { small mammals }\end{array}$ & $\begin{array}{l}\mathrm{r}=0.807-0.981 \\
\mathrm{x}=0.874 ; \mathrm{n}=3\end{array}$ & $\begin{array}{l}r=0.475-0.713 \\
x=0.568 ; n=3\end{array}$ & Shine 1987c \\
\hline Pseudonaja nuchalis & Australia & $\begin{array}{l}\text { Small mammals } \\
\text { and reptiles } \\
\text { (all populations) }\end{array}$ & $\begin{array}{l}\mathrm{r}=0.891-0.988 \\
\mathrm{x}=0.942 ; \mathrm{n}=3\end{array}$ & $\begin{array}{l}r=0.627-0.915 \\
x=0.785 ; n=3\end{array}$ & Shine 1989 \\
\hline Pseudonaja textilis & Australia & $\begin{array}{l}\text { Reptiles and small } \\
\text { mammals (all } \\
\text { populations) }\end{array}$ & $\begin{array}{l}r=0.952-0.994 \\
x=0.972 ; n=3\end{array}$ & $\begin{array}{l}r=0.109-0.711 \\
x=0.387 ; n=3\end{array}$ & Shine 1989 \\
\hline \multicolumn{6}{|l|}{ Viperidae } \\
\hline Agkistrodon piscivorus & USA & $\begin{array}{l}\text { Fish, frogs, snakes } \\
\text { (all populations) }\end{array}$ & $0.942 ; \mathrm{n}=2$ & $0.576 ; \mathrm{n}=2$ & $\begin{array}{l}\text { Himes 2003, Vincent } \\
\text { et al. } 2004\end{array}$ \\
\hline Bitis caudalis & South Africa & $\begin{array}{l}\text { Lizards (all } \\
\text { populations) }\end{array}$ & $\begin{array}{l}r=0.906-0.989 \\
x=0.946 ; n=3\end{array}$ & $\begin{array}{l}r=0.579-0.776 \\
x=0.708 ; n=3\end{array}$ & Shine et al. 1998 \\
\hline Bitis gabonica & Nigeria & $\begin{array}{l}\text { Mammals (all } \\
\text { populations) }\end{array}$ & $\begin{array}{l}r=0.997-0.999 \\
x=0.998 ; n=6\end{array}$ & $\begin{array}{l}r=0.779-0.975 \\
x=0.862 ; n=6\end{array}$ & Luiselli (unpubl.) \\
\hline Bitis nasicornis & Nigeria & $\begin{array}{l}\text { Mammals and frogs } \\
\text { (all populations) }\end{array}$ & $\begin{array}{l}r=0.906-0.989 \\
x=0.965 ; n=6\end{array}$ & $\begin{array}{l}r=0.593-0.976 \\
x=0.778 ; n=6\end{array}$ & Luiselli (unpubl.) \\
\hline $\begin{array}{l}\text { Bothrops atrox } \\
\text { complex }\end{array}$ & $\begin{array}{l}\text { Ecuador, } \\
\text { Peru, } \\
\text { Brazil }\end{array}$ & $\begin{array}{l}\text { Mammals, lizards, } \\
\text { frogs (all populations) }\end{array}$ & $\begin{array}{l}\mathrm{r}=0.836-0.939 \\
\mathrm{x}=0.892 ; \mathrm{n}=4\end{array}$ & $\begin{array}{l}\text { Not calculable from } \\
\text { the original datasets }\end{array}$ & $\begin{array}{l}\text { Duellmann 1978, } \\
\text { Cunha and } \\
\text { Nascimento } 1993, \\
\text { Duellmann and } \\
\text { Mendelson } 1995 \text {, } \\
\text { Nogueira et al. } 2003\end{array}$ \\
\hline $\begin{array}{l}\text { Bothrops neuwiedi } \\
\text { complex }\end{array}$ & Brazil & $\begin{array}{l}\text { Mammals, lizards, } \\
\text { amphibians }\end{array}$ & $0.870 ; \mathrm{n}=2$ & $0.451 ; \mathrm{n}=2$ & $\begin{array}{l}\text { Valdujo et al. 2002, } \\
\text { Hartmann et al. } 2005\end{array}$ \\
\hline $\begin{array}{l}\text { Calloselasma } \\
\text { rhodostoma }\end{array}$ & $\begin{array}{l}\text { Malaysia and } \\
\text { south } \\
\text { Thailand, } \\
\text { Java }\end{array}$ & $\begin{array}{l}\text { Mammals, birds and } \\
\text { reptiles (all } \\
\text { populations) }\end{array}$ & $0.892 ; \mathrm{n}=2$ & $\begin{array}{l}\text { Not calculable from } \\
\text { the original datasets }\end{array}$ & Daltry et al. 1998 \\
\hline Causus maculatus & Nigeria & $\begin{array}{l}\text { Frogs and toads } \\
\text { (all populations) }\end{array}$ & $0.893, \mathrm{n}=2$ & $0.883, \mathrm{n}=2$ & $\begin{array}{l}\text { Luiselli et al. 2004b, } \\
\text { Inyang } 2005\end{array}$ \\
\hline Crotalus atrox & $\begin{array}{l}\text { USA, } \\
\text { Mexico }\end{array}$ & $\begin{array}{l}\text { Rodents (all } \\
\text { populations) }\end{array}$ & $\begin{array}{l}r=0.901-0.978 \\
x=0.956 ; n=3\end{array}$ & $\begin{array}{l}r=0.726-0.903 \\
x=0.822 ; n=3\end{array}$ & $\begin{array}{l}\text { Beavers 1976, } \\
\text { Reynolds and Scott } \\
\text { 1982, Pisani and } \\
\text { Stephenson } 1991\end{array}$ \\
\hline Crotalus horridus & USA & $\begin{array}{l}\text { Rodents (all } \\
\text { populations) }\end{array}$ & $0.989, \mathrm{n}=2$ & $0.776, \mathrm{n}=2$ & $\begin{array}{l}\text { Uhler et al. 1939, } \\
\text { Fitch 1982, } 1999\end{array}$ \\
\hline Crotalus lepidus & USA & $\begin{array}{l}\text { Mammals, lizards, } \\
\text { arthropods (all } \\
\text { populations) }\end{array}$ & $\begin{array}{l}r=0.683-0.967 \\
x=0.776 ; n=3\end{array}$ & $\begin{array}{l}r=0.248-0.339 \\
x=0.294 ; n=3\end{array}$ & $\begin{array}{l}\text { Beaupre 1995, } \\
\text { Holycross et al. } 2002\end{array}$ \\
\hline
\end{tabular}


Table 2. Continued.

\begin{tabular}{|c|c|c|c|c|c|}
\hline Species & $\begin{array}{l}\text { Geographic } \\
\text { origin of } \\
\text { the study } \\
\text { populations }\end{array}$ & Main preys & $\begin{array}{l}\text { Similarity (general } \\
\text { affinity) }\end{array}$ & $\begin{array}{l}\text { Similarity (close } \\
\text { affinity) }\end{array}$ & References \\
\hline Crotalus oreganus & USA & $\begin{array}{l}\text { Mammals and lizards } \\
\text { (all populations) }\end{array}$ & $\begin{array}{l}r=0.726-0.908 \\
x=0.842 ; n=5\end{array}$ & $\begin{array}{l}\text { Not calculable from } \\
\text { the original datasets }\end{array}$ & $\begin{array}{l}\text { Fitch and Twining } \\
\text { 1946, Cunningham } \\
\text { 1959, Diller and } \\
\text { Johnson 1988, } \\
\text { Macartney 1989, } \\
\text { Wallace and Diller } \\
1990\end{array}$ \\
\hline Porthidium godmani & Guatemala & $\begin{array}{l}\text { Arthropods and } \\
\text { mammals (all } \\
\text { populations) }\end{array}$ & $0.979, \mathrm{n}=2$ & $\begin{array}{l}\text { Not calculable from } \\
\text { the original datasets }\end{array}$ & $\begin{array}{l}\text { Campbell and } \\
\text { Solorzano } 1992\end{array}$ \\
\hline Sistrurus catenatus & USA & $\begin{array}{l}\text { Mammals, lizards, } \\
\text { snakes, and } \\
\text { invertebrates } \\
\text { (variable depending } \\
\text { on the populations) }\end{array}$ & $\begin{array}{l}r=0.214-0.987 \\
x=0.650 ; n=6\end{array}$ & $\begin{array}{l}\text { Not calculable from } \\
\text { the original datasets }\end{array}$ & $\begin{array}{l}\text { Greene and Oliver } \\
1965, \text { Keenlyne and } \\
\text { Beer 1973, Hallock } \\
\text { 1991, Holycross and } \\
\text { Mackessy } 2002\end{array}$ \\
\hline Trimeresurus stejnegeri & Taiwan & $\begin{array}{l}\text { Frogs (all } \\
\text { populations) }\end{array}$ & $0.997, \mathrm{n}=2$ & $\begin{array}{l}\text { Not calculable from } \\
\text { the original datasets }\end{array}$ & Creer et al. 2002 \\
\hline Vipera aspis & $\begin{array}{l}\text { Italy, } \\
\text { Switzerland }\end{array}$ & $\begin{array}{l}\text { Rodents (all } \\
\text { populations) }\end{array}$ & $\begin{array}{l}r=0.926-0.996 \\
x=0.948 ; n=4\end{array}$ & $\begin{array}{l}r=0.382-0.843 \\
x=0.678 ; n=4\end{array}$ & $\begin{array}{l}\text { Monney 1996, } \\
\text { Luiselli and Agrimi } \\
\text { 1991, Capizzi and } \\
\text { Luiselli } 1996\end{array}$ \\
\hline Vipera berus & $\begin{array}{l}\text { England, } \\
\text { Denmark, } \\
\text { Poland, } \\
\text { Czech } \\
\text { Republic, } \\
\text { Russia, } \\
\text { Italy }\end{array}$ & $\begin{array}{l}\text { Rodents (all } \\
\text { populations) }\end{array}$ & $\begin{array}{l}r=0.796-0.998 \\
x=0.874 ; n=7\end{array}$ & $\begin{array}{l}r=0.087-0.877 \\
x=0.480 ; n=7\end{array}$ & $\begin{array}{l}\text { Pielowski 1962, Prestt } \\
\text { 1971, Pomianowska- } \\
\text { Pilipiuk 1974, } \\
\text { Kjaergaard 1981, } \\
\text { Luiselli and Anibaldi } \\
\text { 1991, Drobenkov } \\
1995\end{array}$ \\
\hline Vipera ursinii & $\begin{array}{l}\text { France, } \\
\text { Italy }\end{array}$ & $\begin{array}{l}\text { Orthoptera (all } \\
\text { populations) }\end{array}$ & $\begin{array}{l}r=0.936-0.998 \\
x=0.963 ; n=4\end{array}$ & $\begin{array}{l}r=0.792-0.923 \\
x=0.867 ; n=4\end{array}$ & $\begin{array}{l}\text { Agrimi and Luiselli } \\
\text { 1992, Baron 1992, } \\
\text { Filippi and Luiselli } \\
2004\end{array}$ \\
\hline
\end{tabular}

\section{Close affinity}

The mean interpopulation diet similarity value, according to the close affinity criterion $(0.620 \pm 0.230, \mathrm{n}=44)$ was much lesser than that calculated according to the general affinity criterion (see above).

Analysis of variance models (Table 3) showed that: 1) there was no single factor that produced a significant effect on the interpopulation diet variation; 2 ) with regard to the interaction terms between factors, the only significant effect on the interpopulation similarity values was determined by hunting strategy $\times$ venom, with ambushing, venomous species being less variable in their diet composition (Tukey HSD post-hoc test).

\section{Discussion}

This study allowed several generalizations, that should be discussed more in detail.

\section{Why are there minor interpopulation dietary differences in snakes?}

Lots of experimental studies have shown that snakes generally exhibit precise, genetically-determined, species-specific preferences for some prey types (for instance for fish, or for mammals, or for frogs, etc., Arnold 1981, Greene 2001). In addition, snakes are solitary predators (thus can- 


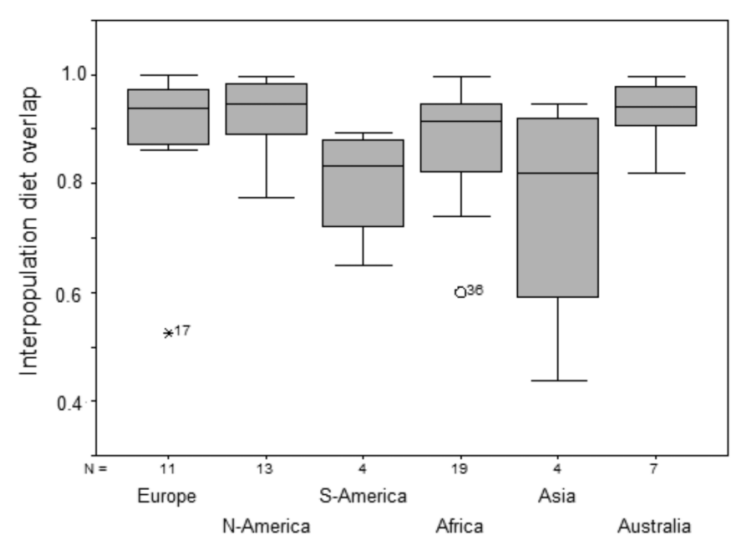

Continent

(c)

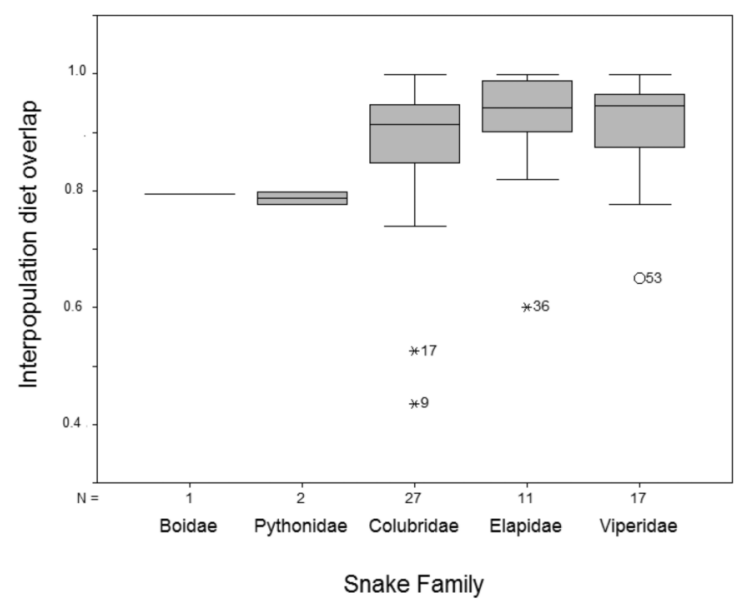

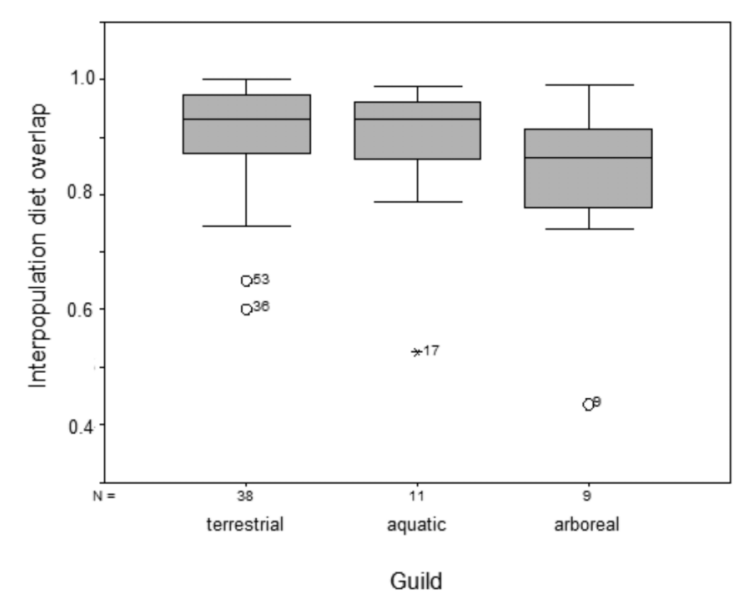

Fig. 2. Means (and dispersion measures) of the interpopulation diet overlap values (general affinity criterion) among continents (a), guilds (b), and snake families (c). Outliers in graphics: $9=$ Elaphe quadrivirgata, $17=$ Natrix maura, $36=$ Notechis scutatus, $53=$ Sistrurus catenatus. $\mathrm{N}=$ number of species.

not benefit of the advantages of group foraging; Clark and Mangel 1986), and are peculiar amongst carnivorous animals because they ingest their prey whole (Greene 2001). Because they cannot tear apart prey items (unlike most vertebrates) and because they eat relatively large prey items (Greene 2001), most snakes are gape-limited predators. Obviously snake skulls are characterized by enormous distensibility (presumably adapted to increase gape, Shine 1991), nonetheless the maximum size of prey ingestible is limited by the size of the snake's head or body (as these two variables are highly correlated) (Savitsky 1983). Given that the majority of the snake species studied to date exhibit low to moderate intraspecific dimorphism in mean body size (but there are some exceptions, see Luiselli 2006a), it can be suggested that most of the species should exhibit low interpopulation variability in prey ingestion ability.
Therefore for most species, given a) the expected minor interpopulation differences in prey size, and b) the genetically-determined prey type preferences, there should be minor interpopulation variations in dietary habits, i.e. exactly the conclusion given by this study. The species which showed the highest interpopulation diet divergence (i.e. the outliers with less overlap value in my analysis) should as a consequence be searched especially among those species with a stronger interpopulation variation in body size. This is verified by data: indeed, the outliers of my analysis showed very remarkable differences in average body size, which are in fact accomplished with dietary composition shifts (Hasegawa and Moriguchi 1989 for Elaphe quadrivirgata and Shine 1987b for Notechis scutatus). This pattern depends on the fact that snakes are gape-limited predators, thus their intraspecific variation in mean body 
Table 3. Comparative results of the effects of the various variables on the interpopulation diet overlap. 1-way ANOVAs were used for measuring the effects of single factors, and GLM - general factorial design for measuring the effects of the interaction terms between independent factors. Effects with significant values are in boldface.

\begin{tabular}{|c|c|c|c|c|}
\hline Source & DF & Mean Square & $\mathrm{F}$ & $\mathrm{p}$ \\
\hline \multicolumn{5}{|l|}{ General affinity criterion } \\
\hline Continent & 5 & $2.603 \mathrm{E}-02$ & 2.063 & 0.085 \\
\hline Climatic region & 1 & $1.313 \mathrm{E}-02$ & 0.951 & 0.334 \\
\hline Guild & 2 & $2.495 \mathrm{E}-02$ & 1.864 & 0.165 \\
\hline Family & 4 & $1.281 \mathrm{E}-02$ & 0.924 & 0.457 \\
\hline Venom & 1 & $3.297 \mathrm{E}-02$ & 2.451 & 0.123 \\
\hline Hunting strategy & 1 & $2.044 \mathrm{E}-03$ & 0.146 & 0.704 \\
\hline Continent $\times$ climatic region & 1 & $7.563 \mathrm{E}-02$ & 6.960 & 0.011 \\
\hline Continent $\times$ guild & 5 & $1.219 \mathrm{E}-02$ & 0.947 & 0.460 \\
\hline Continent $\times$ family & 4 & $2.534 \mathrm{E}-02$ & 2.268 & 0.077 \\
\hline Continent $\times$ venom & 4 & $2.178 \mathrm{E}-02$ & 1.924 & 0.122 \\
\hline Continent $\times$ hunting strategy & 4 & $2.812 \mathrm{E}-02$ & 4.634 & 0.037 \\
\hline Climatic region $\times$ guild & 2 & $5.037 \mathrm{E}-02$ & 4.136 & 0.022 \\
\hline Climatic region $\times$ family & 2 & $9.119 \mathrm{E}-03$ & 0.642 & 0.530 \\
\hline Climatic region $\times$ venom & 1 & $2.657 \mathrm{E}-04$ & 0.020 & 0.889 \\
\hline Climatic region $\times$ hunting strategy & 1 & $2.328 \mathrm{E}-03$ & 0.164 & 0.687 \\
\hline Guild $\times$ family & 4 & $1.249 \mathrm{E}-02$ & 0.887 & 0.479 \\
\hline Guild $\times$ venom & 2 & $2.996 \mathrm{E}-02$ & 2.373 & 0.103 \\
\hline Guild $\times$ hunting strategy & 2 & $1.324 \mathrm{E}-02$ & 0.972 & 0.385 \\
\hline Family $\times$ venom & 1 & $1.521 \mathrm{E}-02$ & 1.080 & 0.304 \\
\hline Family $\times$ hunting strategy & 2 & $3.058 \mathrm{E}-02$ & 2.278 & 0.113 \\
\hline Venom $\times$ hunting strategy & 1 & $5.545 \mathrm{E}-08$ & 0.000 & 0.998 \\
\hline Continent $\times$ hunting strategy $\times$ guild & 2 & $1.577 \mathrm{E}-02$ & 1.396 & 0.261 \\
\hline \multicolumn{5}{|l|}{ Close affinity criterion } \\
\hline Continent & 5 & $7.685 \mathrm{E}-02$ & 1.510 & 0.218 \\
\hline Climatic region & 1 & $2.030 \mathrm{E}-02$ & 0.375 & 0.543 \\
\hline Guild & 2 & 4.586E-02 & 0.854 & 0.433 \\
\hline Family & 4 & $8.269 \mathrm{E}-02$ & 1.644 & 0.183 \\
\hline Venom & 1 & 0.147 & 2.875 & 0.097 \\
\hline Hunting strategy & 1 & $9.056 \mathrm{E}-02$ & 1.727 & 0.196 \\
\hline Continent $\times$ climatic region & - & - & - & - \\
\hline Continent $\times$ guild & 4 & $2.737 \mathrm{E}-02$ & 0.506 & 0.732 \\
\hline Continent $\times$ family & 3 & $2.926 \mathrm{E}-02$ & 0.579 & 0.633 \\
\hline Continent $\times$ venom & 3 & $7.241 \mathrm{E}-03$ & 0.138 & 0.936 \\
\hline Continent $\times$ hunting strategy & 3 & $5.499 \mathrm{E}-02$ & 1.068 & 0.375 \\
\hline Climatic region $\times$ guild & 2 & $7.769 \mathrm{E}-02$ & 1.494 & 0.237 \\
\hline Climatic region $\times$ family & 2 & $2.256 \mathrm{E}-02$ & 0.514 & 0.603 \\
\hline Climatic region $\times$ venom & 1 & $2.720 \mathrm{E}-04$ & 0.005 & 0.943 \\
\hline Climatic region $\times$ hunting strategy & 1 & $2.705 \mathrm{E}-06$ & 0.000 & 0.994 \\
\hline Guild $\times$ family & 3 & $2.602 \mathrm{E}-02$ & 0.152 & 0.927 \\
\hline Guild $\times$ venom & 2 & $2.079 \mathrm{E}-02$ & 0.383 & 0.684 \\
\hline Guild $\times$ hunting strategy & 2 & $2.606 \mathrm{E}-02$ & 0.487 & 0.618 \\
\hline Family $\times$ venom & 1 & $5.810 \mathrm{E}-02$ & 1.138 & 0.293 \\
\hline Family $\times$ hunting strategy & 2 & $9.351 \mathrm{E}-02$ & 1.941 & 0.158 \\
\hline Venom $\times$ hunting strategy & 1 & 0.359 & 8.528 & 0.006 \\
\hline Continent $\times$ hunting strategy $\times$ guild & 1 & $1.169 \mathrm{E}-02$ & 0.202 & 0.657 \\
\hline
\end{tabular}


size (and hence in head size) may strongly condition the maximum size of their prey, and in turn their diet composition.

\section{The effect of continent $\times$ climatic region}

Dietary diversity of a predator is certainly influenced by the diversity of the prey sources (Iwasa et al. 1981, Green 1984, 1990, Stephens and Krebs 1986). Biodiversity (and hence prey diversity) is not equally distributed through the world, but there are particular "hotspots" in tropical regions and in some continents, and in overall there is much more difference in the composition of animal communities between two adjacent areas in the tropics than elsewhere (MacArthur 1972, Rosenzweig 1995, Gaston 2000). Thus, it is predictable that there should be higher interpopulation variations in a predator's diet composition in those continents associated with tropical biodiversity hotspots, i.e. South-America, Asia, and Africa, than in the other continents. The climatic region effect was also significant when associated to guild effect, in that arboreal snakes from the highly species-diverse continents tended to show higher interpopulation diet variation. This may once more depend on the very different patterns in the prey type diversity between tropical and temperate regions also at the level of the arboreal guild. The continental-climatic region effects were not significant under the close affinity criterion, but it may depend on that, for several species, it was impossible to calculate the close affinity values from the original sources (Table 2).

\section{The effects of hunting strategy $\times$ venom, and of hunting strategy $\times$ continent}

Although the hunting strategy taken alone did not have significant effect on snake interpopulation diet variation, it had significant effect when interacted with continent (general affinity criterion) and with venom (close affinity criterion). Taking into consideration both these significant models, it can be generalized that snake species with sitand-wait foraging strategy had significantly less interpopulation variability of diet composition, especially if venomous and if living in Africa, Asia and South America. It is difficult to reconduct this pattern to a clearly predictable hypothesis. I tentatively explain this pattern by two alternative, mutually not-exclusive hypotheses: 1) it may be suggested that sit-and-wait populations of snakes are in general more selective in their habitat/micro-habitat choice, because they depend mainly on camouflage for ambushing with success. Thus, they may encounter more easily the same type of prey in the various localities where they live because the interpopulation variability in habitat type is lesser than in active foragers. 2) Alternatively, it is possible that snakes developed an ambush foraging strat- egy to hunt efficiently on a precise type of prey, and consequently their diet composition evolved in a more "fixed" way than in active foragers. Hypothesis (1) is verified by some sit-and-wait African species that, although with a wide distribution, are found everywhere in the same types of microhabitat (for instance, Bitis nasicornis, see Luiselli 2006c), but is contradicted by other species which show a tremendous variability in habitat and microhabitat selection (e.g. Vipera aspis, Bruno 1985). Hypothesis (2), on the other hand, seems to be more consistent with data coming from other types of predators (Stephens and Krebs 1986), and thus more reliable in order to explain the observed pattern.

Concerning the fact of being venomous, this factor likely determines less interpopulation variability in diet composition because snake venoms have evolved to kill a precise prey type (Daltry et al. 1998, Creer 2000, Creer et al. 2002), and hence it is not convenient for a species that has evolved a costly type of prey-specific killing apparatus to shift considerably from one prey type to another depending on its habitat and locality (Heatwole and Poran 1995). However, there are exceptions to this rule (Daltry et al. 1998), and some highly venomous species (e.g. the African cobras Naja melanoleuca and Naja nigricollis) show very high variability in their prey type preferences in relation to the habitat type (Luiselli et al. 2002), this pattern being in agreement with the fact that they are active foragers (Luiselli et al. 2002).

\section{The non-effect of family}

The taxonomic distribution of snakes along the factors influencing interpopulation diet variation (i.e. continent, climatic region, venom, and hunting strategy) is not equal, but nonetheless shows considerable lability. For instance, 1) venom is associated almost exclusively with species of two families (Elapidae and Viperidae), 2) sit-and-wait strategy is associated essentially with species of the family Viperidae and is very rare in the Colubridae, 3) presence in tropical regions is associated essentially with Boidae, Pythonidae and several Elapidae, and 4) the most speciesrich families (Colubridae, Elapidae, and Viperidae) are broadly distributed across continents. The interaction of these factors with family is therefore never straightforward, and this explains why family did not affect interpopulation diet variation in snakes. Indeed, there are cases of species with very low dietary interpopulation variability in all the three species-rich families (for instance: Hemorrhois hippocrepis for the Colubridae, Dendroaspis jamesoni for the Elapidae, and Bitis gabonica for the Viperidae), and species with high interpopulation diet variability as well (for instance: Elaphe quadrivirgata for the Colubridae, Notechis scutatus for the Elapidae, and Crotalus lepidus for the Viperidae). In any case, it is noteworthy that family is the only factor considered in this paper that did not have 
effect on interpopulation diet variation either alone or in conjunction with other factors. This reinforces even more the evidence that snake family is completely uninfluent for determining whether a species will change its diet composition depending on the various populations.

\section{Future studies}

Although greatly expanding during the recent years, the international literature on snakes is still far from being sufficiently complete to let us understanding all the causes, consequences and correlates of interpopulation dietary variations. Thus, for the future, I would suggest the scientists to study more and more populations of the various snake species from the diet ecology point of view, especially with regard to those species which may exhibit complex patterns of interpopulation dietary variations (for instance, those species which are intraspecifically strongly variable in mean body size or in foraging strategy) or that have been poorly studied up to now. I would also encourage to continue studying the feeding ecology of the well-known species, by adding new populations living in particularly critical environmental contexts (for instance, populations living at the geographic borders of the species' range, or in extreme environmental conditions compared to the usual characteristics of the species' range, etc). It will also be important to study from a interpopulation comparative perspective the relationships between predator size and prey size, which is still a relatively neglected subject in contemporary research of snake feeding ecology.

\section{References}

Agrimi, U. and Luiselli, L. 1992. Feeding strategies of the viper Vipera ursinii ursinii (Reptilia: Viperidae) in the Apennines. - Herpetol. J. 2: 37-42.

Akani, G. C. and Luiselli, L. 2001. Ecological studies on a population of the water snake Grayia smythii in a rainforest swamp of the Niger Delta, Nigeria. - Contrib. Zool. 70: 139-146.

Akani, G. C. et al. 2003. Food habits of the snake Psammophis phillipsi from the continuous rainforest region of southern Nigeria (West Africa). - J. Herpetol. 37: 208-211.

Arnold, S. J. 1981. Behavioral variation in natural populations. I. Phenotypic, genetic and environmental correlations between chemoreceptive responses to prey in the garter snake, Thamnophis elegans. - Evolution 35: 489-509.

Baron, J.-P. 1992. Régime et cycles alimentaires de la Vipère d'Orsini (Vipera ursinii Bonaparte, 1835) au Mont Ventoux, France. - Rev. Ecol. (Terre et Vie) 47: 287-311.

Beaupre, S. J. 1995. Comparative ecology of the mottled rock rattlesnake, Crotalus lepidus, in Big Bend National Park. Herpetologica 51: 45-56.

Beavers, R. A. 1976. Food habits of the western diamondback rattlesnake, Crotalus atrox, in Texas (Viperidae). - Southwest. Nat. 20: 503-515.
Brown, W. S. and Parker, W. S. 1982. Niche dimensions and resource partitioning in a Great Basin Desert snake community. - In: Scott, N. J. Jr (ed.), Herpetological communities. United States Dept of the Interior, pp. 59-81.

Bruno, S. 1985. Vipere d'Italia e d'Europa. - Edagricole.

Camp, C. D., Sprewell, W. D. and Powders, V. N. 1980. Feeding habits of Nerodia taxispilota with comparative notes on the foods of sympatric congeners in Georgia. - J. Herpetol. 14: 301-304.

Campbell, J. A. and Solorzano, A. 1992. The distribution, variation, and natural history of the middle American montane pitviper, Porthidium godmani. - In: Campbell, J. A. and Brodie, E. D. Jr (eds), Biology of the pitvipers. Selva Press, pp. 223-250.

Capizzi, D. and Luiselli, L. 1996. Feeding relationships and competitive interactions between phylogenetically unrelated predators (owls and snakes). - Acta Oecol. 17: 265-284.

Capula, M. and Luiselli, L. 2002. Feeding strategies of Elaphe longissima from contrasting Mediterranean habitats in central Italy. - Ital. J. Zool. 69: 153-156.

Carretero, M. A. 2004. From set menu to a la carte. Linking issues in trophic ecology of Mediterranean lacertids. - Ital. J. Zool. 71 (suppl. 2): 121-133.

Clark, C. and Mangel, M. 1986. The evolutionary advantages of group foraging. - Theor. Popul. Biol. 30: 45-75.

Cooper, W. E. Jr, Burghardt, G. M. and Brown, W. S. 2000. Behavioural responses by hatchling racers (Coluber constrictor) from two geographically distinct populations to chemical stimuli from potential prey and predators. - Amphib.Reptilia 21: 103-116.

Creer, S. 2000. Molecular phylogeography and venom evolution of Trimeresurus stejnegeri in Taiwan. - Unpubl. Ph.D. thesis, Univ. of Wales, Bangor.

Creer, S. et al. 2002. Offshore insular variation in the diet of the Taiwanese bamboo viper Trimeresurus stejnegeri (Schmidt). Zool. Sci. 19: 907-913.

Cunha, O. R. Da and Nascimento, F. P. do 1993. Ofidios de Amazonia. As cobras de regiao leste do Para. - Papeis Avulsos Mus. Par. Emilio Goeldi, n. ser., Zool. 9: 1-191.

Cunningham, J. D. 1959. Reproduction and food of some California snakes. - Herpetologica 15: 17-19.

Daltry, J. C., Wuster, W. and Thorpe, R. S. 1998. Intraspecific variation in the feeding ecology of the crotaline snake Calloselasma rhodostoma in southeast Asia. - J. Herpetol. 32: 198-205.

Diller, L. V. and Johnson, D. R. 1988. Food habits, consumption rates, and predation rates of western rattlesnakes and gopher snakes in southwestern Idaho. - Herpetologica 44: 228-233.

Drobenkov, S. M. 1995. Comparative analysis of diet in sympatric snakes Vipera berus (L.), Natrix natrix (L.), and Coronella austriaca (Laur.). - Ekologija (Moscow) 1995: 222-226.

Duellmann, W. E. 1978. The biology of an equatorial herpetofauna in Amazonian Equador. - Univ. Kans. Mus. Nat. Hist. Misc. Publ. 65: 1-352.

Duellmann, W. E. and Mendelson, J. R. 1995. Amphibians and reptiles from northern Departamento Loreto, Peru: taxonomy and biogeography. - Univ. Kans. Sci. Bull. 55: 329-376.

Filippi, E. 1995. Aspetti dell'ecologia di due comunità di Colubridi e Viperidi (Reptilia, Serpentes) di un'area dell'Italia centrale (Monti della Tolfa, Lazio). - Unpubl. Ph.D. thesis, Univ. of Rome "La Sapienza". 
Filippi, E. and Luiselli, L. 2004. Ecology and conservation of the meadow viper, Vipera ursinii, in three protected mountainous areas in central Italy. - Ital. J. Zool. 71 (suppl. 2): 159161.

Filippi, E. et al. 2005. Comparative food habits and body size of five populations of Elaphe quatuorlineata: the effects of habitat variation and the consequences of intersexual body size dimorphism on diet divergence. - Copeia 2005: 517-525.

Fitch, H. S. 1940. A biogeographical study of the ordinoides artenkreis of garter snakes (genus Thamnophis). - Univ. Calif. Publ. Zool. 44: 1-150.

Fitch, H. S. 1965. An ecological study of the garter snake, Thamnophis sirtalis. - Univ. Kans. Mus. Nat. Hist. Misc. Publ. 62: $1-53$.

Fitch, H. S. 1982. Resources of a snake community in prairiewoodland habitat of northeastern Kansas. - In: Scott, N. J. Jr (ed.), Herpetological communities. United States Dept of the Interior, pp. 83-97.

Fitch, H. S. 1999. A Kansas snake community: composition and changes over 50 years. - Krieger.

Fitch, H. S. and Twining, H. 1946. Feeding habits of the Pacific rattlesnake. - Copeia 1946: 64-71.

Fox, W. 1952. Notes on feeding habits of Pacific Coast garter snakes. - Herpetologica 8: 4-8.

Fukada, H. 1959. Biological studies on the snakes. V. Food habits in the field. - Bull. Kyoto Gakugei Univ. Ser. B 14: 22 28.

Fukada, H. 1992. Snake life history in Kyoto. - Impact Shuppankai, Kyoto.

Gaston, K. J. 2000. Global patterns in biodiversity. - Nature 405: 220-227.

Gibbons, J. W and Dorcas, M. 2004. North American Watersnakes. - Oklahoma Univ. Press.

Green, R. F. 1984. Stopping rules for optimal foragers. - Am. Nat. 123: 30-40.

Green, R. F. 1990. Putting ecology back into optimal foraging theory. - Comm. Theor. Biol. 1: 387-410.

Green, R. F. 2006. A simpler, more general method of finding the optimal foraging strategy for Bayesian birds. - Oikos 112: 274-284.

Greene, H. W. 2001. Snakes: the evolution of mystery in nature. - California Univ. Press.

Greene, H. W. and Oliver, G. V. Jr 1965. Notes on the natural history of the western massasauga. - Herpetologica 21: 225228.

Gregory, P. T. 1978. Feeding habits and diet overlap of three species of garter snakes (Thamnophis) on Vancouver island. Can. J. Zool. 56: 1967-1974.

Gregory, P. T. and Nelson, K. J. 1991. Predation on fish and intersite variation in the diet of common garter snakes, Thamnophis sirtalis, on Vancouver Island. - Can. J. Zool. 69: 988 994.

Gregory, P. T. and Isaac, L. A. 2004. Food habits of the grass snake in southeastern England: is Natrix natrix a generalist predator? - J. Herpetol. 38: 88-95.

Hallock, L. A. 1991. Habitat utilization, diet and behavior of the eastern massasauga (Sistrurus catenatus) in southern Michigan. - Unpubl. M.S. thesis, Michigan State Univ., East Lansing.

Hartmann, M. T. et al. 2005. Feeding habits and habitat use in Bothrops pubescens (Viperidae, Crotalinae) from southern Brazil. - J. Herpetol. 39: 664-667.
Hasegawa, M. and Moriguchi, H. 1989. Geographic variation in food habits, body size, and life history traits of the snakes of the Izu Islands. - In: Matsui, M., Hikida, T. and Goris, R. C. (eds), Current herpetology in east Asia. Herpetological Association of Japan, pp. 414-432.

Heatwole, H. and Poran, N. S. 1995. Resistances of sympatric and allopatric eels to seasnake venoms. - Copeia 1995: 136147.

Himes, J. G. 2003. Diet composition of Nerodia sipedon (Serpentes: Colubridae) and its dietary overlap with, and chemical recognition of Agkistrodon piscivorus (Serpentes: Viperidae). - Amphib.-Reptilia 24: 181-188.

Hirai, T. 2004. Dietary shifts of frog eating snakes in response to seasonal changes in prey availability. - J. Herpetol. 38: 455460.

Holycross, A. T. and Mackessy, S. P. 2002. Variation in the diet of Sistrurus catenatus (massasauga), with emphasis on Sistrurus catenatus edwardsii (desert massasauga). - J. Herpetol. 36: 454-464.

Holycross, A. T. et al. 2002. Diet of Crotalus lepidus klauberi (banded rock rattlesnake). - J. Herpetol. 36: 589-597.

Inyang, M.-A. 2005. Food habits and ecology of arboreal snake populations in southern Nigeria. - Unpubl. dissertation to FIZV, Port Harcourt.

Iwasa, Y., Higashi, M. and Yamamura, N. 1981. Prey distribution as a factor determining the choice of optimal foraging strategy. - Am. Nat. 117: 710-723.

Kadowaki, S. 1992. Food resource overlap between the two sympatric Japanese snakes Elaphe quadrivirgata and Rhabdophis tigrinus. - Jap. J. Ecol. 42: 1-7.

Kadowaki, S. 1996. Ecology of a Japanese snake community: resource use patterns of the three sympatric snakes, Rhabdophis tigrinus, Elaphe quadrivirgata, and Agkistrodon b. blomhoffii. - Bull. Tsukuba Univ. Exper. For. 12: 77-148.

Keenlyne, K. D. and Beer, J. R. 1973. Food habits of Sistrurus catenatus catenatus. - J. Herpetol. 7: 382-384.

King, R. B. 1993. Microgeographic, historical, and size-correlated variation in water snake diet composition. - J. Herpetol. 27: 90-94.

Kjaergaard, J. 1981. A method for examination of stomach contents in live snakes and some information on feeding habits in common viper (Vipera berus) in Denmark. - Natura Jutlandica 19: 45-48.

Lekunze, L. M., Ezealor, A. U. and Aken'Ova, T. 2001. Prey groups in the pellets of the barn owl Tyto alba (Scopoli) in the Nigerian savanna. - Afr. J. Ecol. 39: 38-44.

Luiselli, L. 2003. Do snakes exhibit shifts in feeding ecology associated with the presence or absence of potential competitors? A case study from tropical Africa. - Can. J. Zool. 81: 228236.

Luiselli, L. 2006a. Resource partitioning and interspecific competition in snakes: the search for general geographical and guild patterns. - Oikos 113: 385-404.

Luiselli, L. 2006b. Interspecific relationships between two species of sympatric Afrotropical water snake in relation to a seasonally fluctuating food resource. - J. Trop. Ecol. 22: 91-100.

Luiselli, L. 2006c. Site occupancy and density of sympatric Gaboon viper (Bitis gabonica) and nose-horned viper (Bitis nasicornis). - J. Trop. Ecol. 22, in press.

Luiselli, L. and Agrimi, U. 1991. Composition and variation of the diet of Vipera aspis francisciredi in relation to age and reproductive stage. - Amphib.-Reptilia 12: 137-144. 
Luiselli, L. and Anibaldi, C. 1991. The diet of the adder (Vipera berus) in two alpine environments. - Amphib.-Reptilia 12: 214-217.

Luiselli, L. and Rugiero, L. 1991. Food niche partitioning by water snakes (genus Natrix) at a freshwater environment in central Italy. - J. Freshwater Ecol. 6: 439-444.

Luiselli, L. and Angelici, F.M. 1998. Sexual size dimorphism and natural history traits are correlated with intersexual dietary divergence in royal pythons (Python regius) from the rainforests of southeastern Nigeria. - Ital. J. Zool. 65: 183185.

Luiselli, L., Capula, M. and Shine, R. 1996. Reproductive output, costs of reproduction, and ecology of the smooth snake, Coronella austriaca, in the eastern Italian Alps. - Oecologia 106: 100-110.

Luiselli, L., Capula, M. and Shine, R. 1997. Food habits, growth rates and reproductive biology of grass snakes, Natrix natrix (Colubridae) in the Italian Alps. - J. Zool. Lond. 241: 371380.

Luiselli, L., Akani, G. C. and Barieenee, I. F. 1998a. Observations on habitat, reproduction and feeding of Boiga blandingi (Colubridae) in south-eastern Nigeria. - Amphib.-Reptilia 19: 430-436.

Luiselli, L., Akani, G. C. and Capizzi, D. 1998b. Food resource partitioning of a community of snakes in a swamp rainforest of south-eastern Nigeria. - J. Zool. Lond. 246: 125-133.

Luiselli, L., Angelici, F. M. and Akani, G. C. 1999-2000. Reproductive ecology and diet of the Afrotropical tree snake Rhamnophis aethiopissa (Colubridae). - Herpetol. Nat. Hist. 7: 153-161.

Luiselli, L., Akani, G. C. and Angelici, F. M. 2000a. Observations on the biology of the African emerald snake Gastropyxis smaragdina (Schlegel, 1837), in southern Nigeria. - Herpetozoa 13: 3-14.

Luiselli, L., Angelici, F. M. and Akani, G. C. 2000b. Large elapids and arboreality: the ecology of Jameson's green mamba (Dendroaspis jamesoni) in an Afrotropical forested region. Contrib. Zool. 69: 147-155.

Luiselli, L. et al. 2001a. Geographic variation in the diet composition of a secretive Mediterranean colubrid snake: Coronella girondica from Spain and Italy. - Ital. J. Zool. 68: 57-60.

Luiselli, L., Angelici, F. M. and Akani, G. C. 2001b. Food habits of Python sebae in suburban and natural habitats. - Afr. J. Ecol. 39: 116-118.

Luiselli, L., Angelici, F. M. and Akani, G. C. 2002. Comparative feeding strategies and dietary plasticity of the sympatric cobras Naja melanoleuca and Naja nigricollis in three diverging Afrotropical habitats. - Can. J. Zool. 80: 55-63.

Luiselli, L. et al. 2004a. Local distribution, habitat use, and diet of two supposed 'species' of the Psammophis 'phillipsi' complex (Serpentes: Colubridae), sympatric in southern Nigeria. - Amphib.-Reptilia 25: 415-423.

Luiselli, L. et al. 2004b. Aspects of the ecology of a population of Causus maculatus (Reptilia: Viperidae) from southern Nigeria. - Amphibia-Reptilia 25: 99-104.

Luiselli, L., Filippi, E. and Capula, M. 2005. Geographic variation in diet composition of the grass snake (Natrix natrix) along the mainland and an island of Italy: the effects of habitat type and interference with potential competitors. - Herpetol. J. 15: 221-230.

MacArthur, R. H. 1972. Geographical ecology: patterns in the distribution of species. - Harper and Row.
MacArthur, R. H. and Levins, R. 1967. The limiting similarity, convergence and divergence of coexisting species. - Am. Nat. 101: 377-385.

MaCartney, J. M. 1989. Diet of the northern Pacific rattlesnake, Crotalus viridis oreganos, in British Columbia. - Herpetologica 45: 299-304.

Madsen, T. 1983. Growth rates, maturation and sexual size dimorphism in a population of grass snakes, Natrix natrix in southern Sweden. - Oikos 40: 277-282.

May, R. M. 1975. Some notes on estimating the competition matrix, a. - Ecology 56: 737-741.

McCullagh, P. and Nelder, J. A. 1989. Generalized linear models. - Chapman and Hall.

McCullagh, P. and Searle, S. R. 2002. Generalized, linear, and mixed models. - Wiley.

Monney, J.-C. 1996. Biologie comparée de Vipera aspis L. et de Vipera berus L. (Reptilia, Ophidia, Viperidae) dans une station des Prealpes Bernoises. - Unpubl. Ph.D. thesis, Univ. of Neuchatel.

Mushinsky, H. R. 1987. Foraging ecology. - In: Seigel, R. A., Collins, J. T. and Novak, S. S. (eds), Snakes: ecology and evolutionary biology. MacMillan, pp. 302-334.

Nogueira, C., Sawaya, R. J. and Martins, M. 2003. Ecology of the pitviper, Bothrops moojeni, in the Brazilian Cerrado. - J. Herpetol. 37: 653-659.

Olsson, O. and Brown, J. S. 2006. The foraging benefits of information and the penalty of ignorance. - Oikos 112: 260273.

Pianka, E. R. 1986. The ecology and natural history of desert lizards. - Princeton Univ. Press.

Pielowski, Z. 1962. Untersuchungen über die Ökologie der Kreuzotter (Vipera berus L.). - Zool. Jahr. 89: 479-500.

Pisani, G. R. and Stephenson, B. R. 1991. Food habits in Oklahoma: Crotalus atrox in fall and early spring. - Trans. Kansas Acad. Sci. 94: 137-141.

Pleguezuelos, J. M. and Moreno, M. 1990. Alimentacion de Coluber hippocrepis en el SE de la Peninsula Ibérica. - Amphib.Reptilia 11: 325-337.

Pleguezuelos, J. M. and Fahd, S. 2004. Body size, diet and reproductive ecology of Coluber hippocrepis in the Rif (northern Morocco). - Amphib.-Reptilia 25: 287-302.

Politano, E. 1985. Studi propedeutici sulla fauna del Delta del Fiume Niger in Nigeria. - ENI Press, Milan.

Politano, E. 1998. A study of the fauna (Amphibia, Reptilia, Aves, Mammalia) of the Port Harcourt region in the Niger Delta, Nigeria, and assessment of the effects of the LNG Bonny project on the environmental conditions of the whole region. - Aquater Press, San Lorenzo in Campo.

Pomianowska-Pilipiuk, I. 1974. Energy bilance and food requirements of adult vipers Vipera berus berus (L.). - Ekol. Polska 22: 195-211.

Prestt, I. 1971. An ecological study of the viper Vipera berus in southern Britain. - J. Zool. Lond. 164: 373-418.

Quick, J. S. et al. 2005. Recent occurrence and dietary habits of Boa constrictor on Aruba, West Indies. - J. Herpetol. 39: 304-307.

Ray, J. C. and Sunquist, M. E. 2001. Trophic relations in a community of African rainforest carnivores. - Oecologia 127: 395-408.

Reading, C. J. and Davies, J. L. 1996. Predation by grass snakes (Natrix natrix) at a site in southern England. - J. Zool. Lond. 239: 73-82. 
Reynolds, R. P. and Scott, N. J. Jr 1982. Use of a mammalian resource by a Chihuahuan snake community. - In: Scott, N. J. Jr (ed.), Herpetological communities. US Dept of the Interior, pp. 99-118.

Rodriguez-Robles, J. A. 2002. Feeding ecology of North American gopher snakes (Pituophis catenifer). - Biol. J. Linn. Soc. 77: 165-183.

Rosenzweig, M. L. 1995. Species diversity in space and time. Cambridge Univ. Press.

Rugiero, L. and Luiselli, L. 1995. Food habits of the snake Coluber viridiflavus in relation to prey availability. - Amphib.Reptilia 16: 407-411.

Rugiero, L. et al. 1995. Food habits of Mediterranean populations of the smooth snake (Coronella austriaca). - Herpetol. J. 5: 316-318.

Rugiero, L. et al. 2000. Life-history and diet of two populations of Natrix maura (Reptilia, Colubridae) from contrasted habitats in Sardinia. - Misc. Zool. 23: 41-51.

Rugiero, L., Capizzi, D. and Luiselli, L. 2002. Interactions between sympatric snakes, Coluber viridiflavus and Elaphe longissima: are there significant inter-annual differences in coexistence patterns? - Ecol. Medit. 28: 75-91.

Saint Girons, H. 1980. Modifications sélectives du régime des vipères (Reptilia: Viperidae) lors de la croissance. - Amphib.Reptilia 1: 127-136.

Santos, X. and Llorente, G.A. 1998. Sexual and size-related differences in the diet of the snake Natrix maura from the Ebro Delta, Spain. - Herpetol. J. 8: 161-165.

Savitsky, A. H. 1983. Coadapted character complexes among snakes: fossoriality, piscivory, and durophagy. - Am. Zool. 23: 397-409.

Schoener, T. W. 1971. Theory of feeding strategies. - Annu. Rev. Ecol. Syst. 11: 369-404.

Shetty, S. and Shine, R. 2002. Sexual divergence in diets and morphology in Fijian sea snakes, Laticauda colubrina (Laticaudidae). - Aust. Ecol. 27: 77-84.

Shine, R. 1980. Ecology of eastern Australian whipsnakes of the genus Demansia. - J. Herpetol. 14: 381-389.

Shine, R. 1981. Ecology of Australian elapid snakes of the genera Furina and Glyphodon. - J. Herpetol. 15: 219-224.

Shine, R. 1982. Ecology of the Australian elapid snake Echiopsis curta. - J. Herpetol. 16: 388-393.

Shine, R. 1984. Reproductive biology and food habits of the Australian elapid snakes of the genus Cryptophis. - J. Herpetol. 18: 33-39.

Shine, R. 1987a. Food habits and reproductive biology of Australian snakes of the genus Hemiaspis (Elapidae). - J. Herpetol. 21: 71-74.
Shine, R. 1987b. Ecological comparisons of island and mainland populations of Australian tigersnakes (Notechis: Elapidae). Herpetologica 43: 233-240.

Shine, R. 1987c. The evolution of viviparity: ecological correlates of reproductive mode within a genus of Australian snakes (Pseudechis: Elapidae). - Copeia 1987: 551-563.

Shine, R. 1989. Constraints, allometry and adaptation: food habits and reproductive biology of Australian brownsnakes (Pseudonaja: Elapidae). - Herpetologica 45: 195-207.

Shine, R. 1991. Intersexual dietary divergence and the evolution of sexual dimorphism in snakes. - Am. Nat. 138: 103122.

Shine, R. and Bonnet, X. 2000. Snakes: a new 'model organism' in ecological research? - Trends Ecol. Evol. 15: 221-222.

Shine, R. et al. 1998. Reproductive biology and food habits of horned adders, Bitis caudalis (Viperidae), from Southern Africa. - Copeia 1998: 391-401.

Shine, R. et al. 2002. Accidental altruism in insular pit-vipers (Gloydius shedaoensis, Viperidae). - Evol. Ecol. 16: 541548.

Sironi, M. et al. 2000. Dietary habits of Boa constrictor occidentalis, in the Cordoba Province, Argentina. - Amphib.-Reptilia 21: 226-232.

Stephens, D. W. and Krebs, J. R. 1986. Foraging theory. - Princeton Univ. Press.

Tanaka, H. and Ota, K. 2002. Natural history of two colubrid snakes, Elaphe quadrivirgata and Rhabdophis tigrinum, on Yakushima island, southwestern Japan. - Amphib.-Reptilia 23: 323-331.

Uhler, F. M., Cottam, C. and Clarke, T.E. 1939. Food of snakes of the George Washington National Forest, Virginia. - In: Transactions of the Fourth North American Wildlife Conference, pp. 605-622.

Valdujo, P. H., Nogueira, C. and Martins, M. 2002. Ecology of Bothrops neuwiedi pauloensis (Serpentes: Viperidae: Crotalinae) in the Cerrado of central and southeastern Brazil. - J. Herpetol. 36: 169-176.

Vincent, S. E., Herrel, A. and Irschik, D. J. 2004. Sexual dimorphism in head shape and diet in the cottonmouth snake ( $A g$ kistrodon piscivorus). - J. Zool. (Lond.) 264: 53-59.

Wallace, R. L. and Diller, L. V. 1990. Feeding ecology of the rattlesnake Crotalus viridis oreganus in northern Idaho. - J. Herpetol. 24: 246-253.

Zimmermann, P. and Fachbach, G. 1996. Verbreitung und Biologie der Wurfelnatter, Natrix tessellata tessellata (Laurenti, 1768), in der Steiermark (Osterreich). - Herpetozoa 7: 99124. 